\title{
Differential expression of salivary glycoproteins in aggressive and chronic periodontitis
}

\author{
Daniela de Morais ROCHA ${ }^{1}$, Elton Gonçalves ZENÓBIO², Thomas VAN DYKE ${ }^{3}$, Karine Simões SILVA ${ }^{4}$, Fernando \\ Oliveira COSTA ${ }^{5}$, Rodrigo Villamarim SOARES ${ }^{2}$
}

\begin{abstract}
1- DDS, MSc, School of Dentistry, Pontifical Catholic University of Minas Gerais, Belo Horizonte, MG, Brazil.
2- DDS, DSc, Adjunct Professor, School of Dentistry, Pontifical Catholic University of Minas Gerais, Belo Horizonte, MG, Brazil.

3- DDS, PhD, Adjunct Professor, Department of Periodontology and Oral Biology, Director, Postgraduate Periodontology, Director, Clinical Research Center, Goldman School of Dental Medicine, Boston University, Boston, USA.

4- Undergraduate student, School of Dentistry, Pontifical Catholic University of Minas Gerais, Belo Horizonte, MG, Brazil.

5- DDS, DSc, Adjunct Professor, Department of Oral Surgery and Pathology, School of Dentistry, Pontifical Catholic University of Minas Gerais, Belo Horizonte, MG, Brazil.
\end{abstract}

Corresponding address: Prof. Dr. Rodrigo Villamarim Soares - Programa de Mestrado em Clínicas Odontológicas - PUC Minas - Av. Dom José Gaspar, 500, Bairro Coração Eucarístico - Belo Horizonte - MG - Brasil - 30535-610 - Phone: + 55-31-3319-4414 - Fax: + 55-31-3319-4415 - e-mail: soaresrv@pucminas.br

Received: June 09, 2010 - Modification: March 02, 2011 - Accepted: April 27, 2011

\section{ABSTRACT}

\begin{abstract}
O bjectives: The aim of this study was to compare the pattern of secretion and the expression of mucin glycoprotein-2 (MG2) and lactoferrin in individuals with or without periodontitis. Material and Methods: Five individuals with aggressive periodontitis (APG), 5 with generalized chronic periodontitis (CPG) and 5 without periodontitis (CG) were enrolled after informed consent. Non-stimulated and stimulated submandibular and sublingual saliva was collected and samples analyzed by Western blot probed with specific antibodies. Results: Stimulated and non-stimulated salivary flow rates did not differ among groups. Western blot analysis revealed that stimulation led to: an increase in MG2 expression in all groups, and to lactoferrin expression in APG and CPG. In non-stimulated saliva, CG exhibited the highest expression of both glycoproteins. In stimulated saliva, CG exhibited the highest expression of MG2, whereas APG the highest of lactoferrin. Conclusions: The pattern of secretion of MG2 and lactoferrin in health and disease is complex. Although the present study analyzed samples from a limited number of participants, the reduced expression of MG2 and lactoferrin in APG and CPG under non-stimulated condition, the predominant circumstance of salivary secretion during the day, suggests that these salivary constituents may play a role in the etiopathogenesis of these diseases.
\end{abstract}

Key words: Submandibular gland. Sublingual gland. Saliva. Innate immunity. Periodontitis. Glycoproteins.

\section{INTRODUCTION}

Periodontal diseases (PD) are chronic inflammatory disorders encompassing destructive and nondestructive diseases of the periodontal supporting tissues of the teeth. Aggressive periodontitis is characterized by severe and rapid loss of periodontal attachment often commencing at or after puberty. Chronic periodontitis is a common disease that is prevalent among adults and seniors ${ }^{5,12}$. Even though the diagnosis and classification of PD is essentially based on clinical parameters, new auxiliary diagnostic tools based on the analysis of saliva and gingival crevicular fluid have been studied and developed 6 . In particular, saliva has been extensively studied in relation to PD since it can be easily collected and analyzed ${ }^{22}$.

Saliva is necessary for the maintenance of oral health, and the unique properties of this fluid are derived in large part from the proteins that are present. In the oral cavity, salivary proteins participate in formation of the acquired enamel pellicle, occur in the biofilm covering oral surfaces, initiate digestion, promote agglutination and clearance of bacteria, and protect oral tissues against noxious compounds produced by various microorganisms ${ }^{30}$.

In particular, mucin glycoprotein-2 (MG2) 
was reported to be present in pellicle formed on cementum surfaces ${ }^{11}$, to exhibit candidacidal activity $^{24}$ and to kill the periodontal pathogen Aggregatibacter actinomycetemcomitans ${ }^{26}$. Salivary lactoferrin removes free iron in fluids decreasing the availability of this metal to microbes ${ }^{27}$. In addition, lactoferrin exhibits antibacterial, antimycotic, antiviral, and anti-inflammatory activity ${ }^{35}$. Thus, MG2 and lactoferrin are active components of the innate immune system in the oral environment.

Several studies reported that the levels of distinct salivary proteins are altered in individuals with PD ${ }^{10,14-19,32}$. Therefore, the present study was undertaken to investigate and compare the pattern of secretion and the expression of MG2 and lactoferrin in individuals with aggressive periodontitis, chronic periodontitis and without periodontitis.

\section{MATERIAL AND METHODS}

\section{Participant selection}

During a 12-month period, individuals that were referred to the authors' department for treatment and volunteered to participate in this study were screened. Exclusion criteria for the study included: pregnancy; use of antibiotics within the past 3 months; individuals with systemic debilitating diseases or conditions that could affect periodontal status (e.g. diabetes); smokers and former smokers; recent trauma. Additionally, individuals that had aggressive or chronic periodontitis and had received periodontal treatment within the previous year were excluded in order to observe the expression of the salivary glycoproteins MG2 and lactoferrin in the inflammatory context of periodontitis.

Individuals meeting inclusion criteria received a complete periodontal exam that included assessment of probing depth, probing attachment level and recession, evaluation of bleeding and/or suppuration on probing, radiographic examination and complete anamnesis. Probing depths were measured at six sites per tooth (mesio-, mid-, disto-vestibular/palatal or lingual). The specific inclusion criteria used to differentiate the 3 groups were based on previous publications ${ }^{2-5}$ including:

Aggressive periodontitis group (APG): (I) localized aggressive periodontitis - periodontal damage being localized to permanent first molars and incisors; generalized aggressive periodontitis: (II) generalized interproximal attachment loss affecting at least 3 permanent teeth other than the permanent first molars and incisors; (III) clinical attachment loss $(\mathrm{CAL}) \geq 5 \mathrm{~mm}$; probing depths $\geq 6$ $\mathrm{mm}$. Generalized chronic periodontitis group (CPG): (I) more than $30 \%$ of sites involved; moderate: CAL 3-4 mm; probing depths 4-6 $\mathrm{mm}$; severe: CAL $\geq 5$ $\mathrm{mm}$; probing depths $\geq 6 \mathrm{~mm}$. Control group (CG): all sites with probing depths $<4 \mathrm{~mm}$.

Each group had 5 individuals ( 2 males and 3 females) with age ranges of 19-28 years for APG, 36-50 years for CPG, 21-50 years for CG and as expected ${ }^{33}$ individuals from APG exhibited a lower age range. This study was independently reviewed and approved by the local Research Ethics Committee, informed consent was obtained from all individuals prior to their participation and subjects' rights were protected at all times.

\section{Saliva collection}

Non-stimulated and stimulated submandibular and sublingual saliva (SMSL) was collected from subjects between 10:00 a.m. and 11:00 a.m. Subjects were asked to refrain from eating or drinking two h prior to collection. Subjects rinsed with water, and SMSL samples were collected with a custom-fitted device as described previously ${ }^{20}$.

The first $4 \mathrm{~min}$ of non-stimulated SMSL were discarded in order to avoid possible interference of the device dead volume. Four consecutive two-minute samples collected in separate $1.5 \mathrm{~mL}$ centrifuge tubes and subsequently four consecutive two-minute samples of stimulated SMSL were collected in separate $15 \mathrm{~mL}$ Falcon screw cap tubes. Gustatory stimulation of stimulated samples was induced by placing lemon fruit flavored candies on the tongue. Tubes of non-stimulated SMSL were labeled T1, T2, T3 and T4. Tubes of stimulated SMSL were labeled T5, T6, T7 and T8. All samples were kept on ice during the collection procedure, the flow rate from each subject was recorded and samples were frozen at $-20^{\circ} \mathrm{C}$ until used.

\section{Gel electrophoresis}

SMSL samples were thawed, and for each subject, equal volumes $(50 \mu \mathrm{L})$ of non-stimulated and stimulated SMSL were lyophilized, taken up in $15 \mu \mathrm{L}$ of sample buffer, heated at $95^{\circ} \mathrm{C}$ for $5 \mathrm{~min}$ and subjected to sodium dodecylsulfate polyacrylamide gel electrophoresis (SDS-PAGE) on $7.5 \%$ gels.

\section{Western blots}

Proteins in gels were electrophoretically transferred to nitrocellulose membranes (Protran, Schleicher and Schuell, Keene, NH, USA) in 25 $\mathrm{mM}$ Tris- $\mathrm{HCl}, \mathrm{pH} 8.3$, containing $192 \mathrm{mM}$ glycine and $20 \%$ methanol at 100 volts for $1 \mathrm{~h}$ at room temperature. Blots were probed with rabbit polyclonal antibodies directed against MG2 or lactoferrin. Briefly, blots were equilibrated in 10 $\mathrm{mM}$ Tris- $\mathrm{HCl}$, pH 8.0 containing $150 \mathrm{mM} \mathrm{NaCl}$ and $0.1 \%$ Tween-20 (TBST) for 5 min and blocked with $5 \%$ non-fat, dried milk in TBST overnight at room temperature. After blocking, blots were washed three times with TBST for $5 \mathrm{~min}$. The specificity of 
the anti-MG2 antibody has been demonstrated in previous studies ${ }^{25,29}$, and this antibody was diluted 1:1000. The anti-lactoferrin antibody (Sigma, St. Louis, MO, USA) was diluted 1:2,000. Antibodies were diluted in $1 \%$ milk/TBST and incubated for 1 $\mathrm{h}$ at room temperature. After washing, blots were incubated with the second antibody, which was anti-rabbit IgG coupled to alkaline phosphatase (Promega, Madison, WI, USA) diluted 1:7,500 in $1 \%$ milk/TBST for $1 \mathrm{~h}$ at room temperature. Blots were washed three times in TBST for 5 min and color development was carried out with 5-bromo4-chloro-3-indolyl-phosphate (BCIP) and nitro blue tetrazolium (NBT) according to the manufacturer's (Promega) instructions.

\section{Statistical analysis}

Comparison of the effect of non-stimulated and stimulated conditions on SMSL flow rate was tested for statistical significance $(a=0.05)$ by a two-way repeated-measures analysis of variance (ANOVA; post hoc analysis - Fisher's exact test). The statistical analysis was performed using StatView 4.5 (Abacus Concepts Inc., Berkeley, CA, USA).

\section{RESULTS}

\section{SMSL flow rate}

As expected, the observed flow rate values obtained at rest (T1-T4) were lower than stimulated (T5-T8) conditions in all groups. The mean value for flow rates under non-stimulated conditions was $0.46 \pm 0.18 \mathrm{~mL} / \mathrm{min}$ for $\mathrm{APG}, 0.30 \pm 0.20$ for $\mathrm{CPG}$ and $0.47 \pm 0.31$ for $C G$. Under stimulated conditions, it was $1.79 \pm 0.92 \mathrm{ml} / \mathrm{min}$ for $A P G, 1.47 \pm 0.38$ $\mathrm{mL} / \mathrm{min}$ for $\mathrm{CPG}$ and $1.87 \pm 0.99 \mathrm{~mL} / \mathrm{min}$ for CG. Stimulation significantly raised salivary volumes in all groups (APG - $p=0.01 ; C P G-p=0.03 ; C G$ $\mathrm{p}=0.01)$; no significant time effect was observed for non-stimulated or stimulated conditions, and the observed flow rates under both conditions did not differ among groups.

\section{Analysis of the secretion patterns}

MG2 immunoreactive bands from APG, CPG and CG exhibited a weak immunoreactive signal under non-stimulated conditions whereas upon gustatory stimulation, the intensity of the immunoreactive signal increased (Figure $1 \mathrm{~A}$ ). Blots probed with anti-lactoferrin antibodies revealed a difference in banding pattern among groups (Figure 1B); individuals from APG and CPG had an increase in expression upon gustatory stimulation, whereas individuals from CG exhibited a decrease.

\section{Comparison of glycoprotein expression \\ Comparison of expression among groups} under non-stimulated conditions revealed that
CG exhibited the highest expression of MG2 and lactoferrin (Figures $2 \mathrm{~A}$ and $2 \mathrm{~B}$ ). APG showed the lowest levels of MG2, and CPG of lactoferrin (Figures $2 \mathrm{~A}$ and $2 \mathrm{~B}$ ). Comparison of expression among groups under stimulated conditions showed that CG exhibited the highest expression of MG2, whereas APG the highest of lactoferrin (Figures 2C and 2D). Again, APG showed the lowest levels of MG2, and

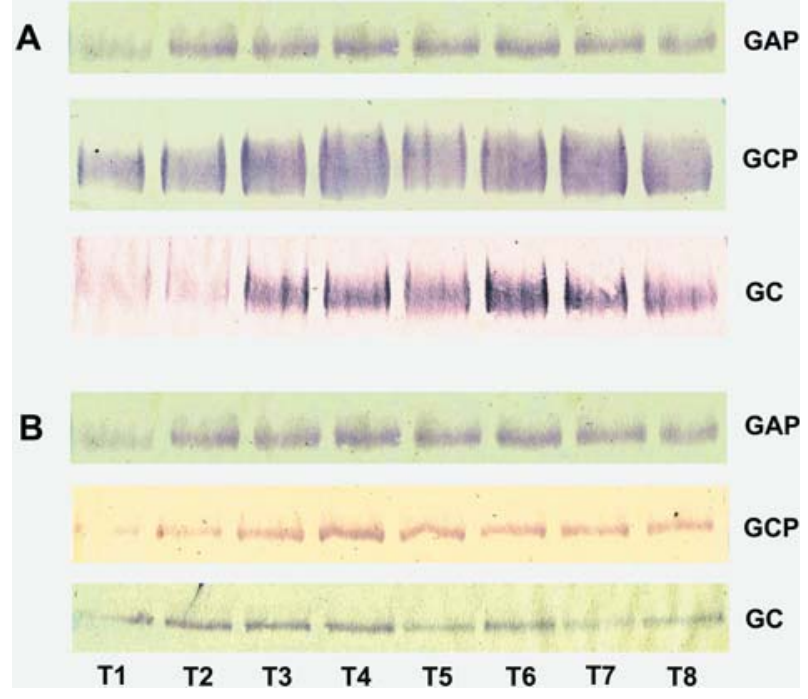

Figure 1- Pattern of secretion of salivary glycoproteins. A - Representative Western blots of MG2 pattern observed in all individuals from each group. B - Representative Western blots of lactoferrin pattern observed in all individuals from each group. T1-T4 (non-stimulated submandibular and sublingual saliva-SMSL) and T5-T8 (stimulated submandibular and sublingual saliva-SMSL). APG-Aggressive periodontitis group; CPG-Generalized chronic periodontitis group; CG-Control group

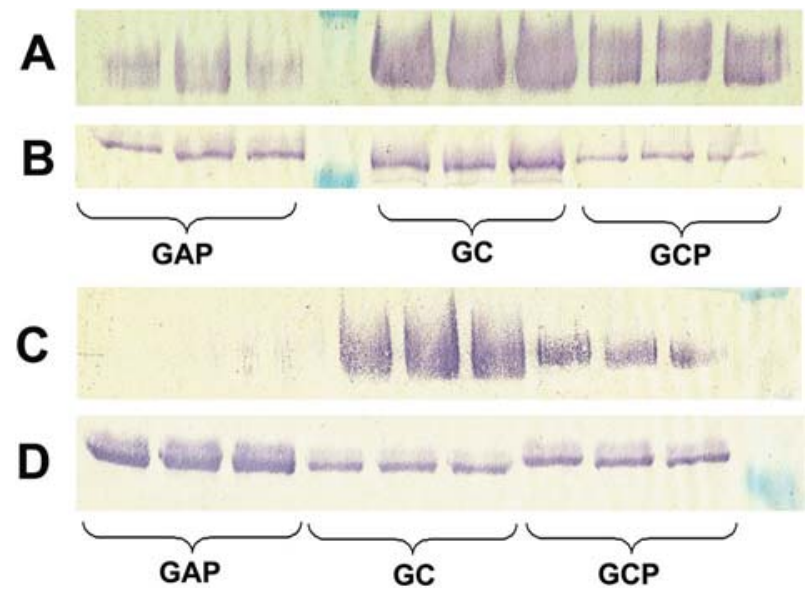

Figure 2- Comparison of glycoproteins expression under non-stimulated (A, B) or stimulated (C, D) conditions. A-MG2 Western blot; B-Lactoferrin Western blot; C-MG2 Western blot; D-Lactoferrin Western blot; APGAggressive periodontitis group; CPG-Generalized chronic periodontitis group; CG-Control group 
CPG of lactoferrin (Figures 2C and 2D).

\section{DISCUSSION}

The infectious nature of the periodontitis is related to specific microbiota as well as to the expression of particular inflammatory markers ${ }^{9,28,34}$. Additionally, initiation and progression of periodontal infections is clearly modified by local and systemic conditions (risk factors), such as diabetes mellitus and cigarette smoking, as well as potentially important risk indicators including stress ${ }^{13}$. Salivary proteins are not included in the category of risk indicators, but this concept is being explored.

Investigation of salivary proteins in individuals with periodontitis may be useful to enhance the knowledge of their role in this population. Statistical analysis did not reveal flow rate differences among groups, stimulated or non-stimulated. Therefore, we decided to analyze equal volumes $(50 \mu \mathrm{L})$ of SMSL by immunoblotting, instead of equal amounts of protein.

It has been previously reported that MG2 can agglutinate several pathogens from the oral cavity ${ }^{8,23,31}$, and in addition, interact and kill the periodontal pathogen $A$. actinomycetemcomitans ${ }^{26}$. In a previous study, the concentration of MG2 was determined in stimulated whole saliva of subjects suffering from $A$. actinomycetemcomitans associated $\mathrm{PD}^{14}$. It has been reported that MG2 output in the diseased subjects was decreased at least by a factor three in comparison to periodontally healthy subjects and that this low concentration of MG2 suggested a decline in mucin defense and consequently, a higher susceptibility to oral infections.

In the present study, blotting analysis revealed that all groups showed an increase of MG2 expression upon stimulation revealing a similar pattern (Figures $1 \mathrm{~A}$ and $1 \mathrm{~B}$ ). A previous study that analyzed the pattern of secretion of MG2 in samples from individuals without periodontitis ${ }^{7}$ reported that PAS stained gels did not reveal differences in MG2 secretion patterns upon stimulation. However, Western blots indicated that MG2 expression was enhanced after stimulation; a result that is in agreement with the observed pattern in the present study for CG.

It was also observed that individuals without periodontitis exhibited the highest expression of this small salivary mucin under specific conditions (Figures $2 \mathrm{~A}$ and $2 \mathrm{C}$ ). The reduced output of this salivary mucin in individuals with aggressive periodontitis observed on blots (Figures $2 \mathrm{~A}$ and $2 \mathrm{C}$ ) is similar to the one described ${ }^{14}$. Since in chronic periodontitis the destruction of the periodontal ligament and loss of the adjacent supporting bone usually displays a slow to moderate rate of progression, we hypothesize that the intermediate expression of MG2 observed in individuals belonging to this group (Figures $2 \mathrm{~A}$ and $2 \mathrm{C}$ ) could in part contribute to decrease the disease progression rate in comparison to aggressive periodontitis.

Salivary lactoferrin is a single chain ironbinding glycoprotein that binds ferric ions and possesses antibacterial, antimycotic, antiviral and anti-inflammatory properties ${ }^{35}$. It also exhibits the ability to penetrate bacterial cell membranes ${ }^{36}$. The impact of a surgical periodontal treatment in the concentration of this glycoprotein in nonstimulated and stimulated whole saliva samples from individuals with chronic periodontitis was analyzed previously ${ }^{21}$. It was reported that the concentrations decreased significantly in both non-stimulated and stimulated samples after the surgical treatment, suggesting that lactoferrin may be suitable for monitoring periodontal treatment results.

Another study evaluated lactoferrin levels from individuals with localized aggressive periodontitis before and after periodontal therapy. Among other samples, paraffin-stimulated whole saliva was obtained from these individuals and from periodontally healthy controls. Differences between groups and with respect to periodontal therapy were not observed in lactoferrin concentrations in whole saliva ${ }^{32}$.

Concentrations and output of lactoferrin were also determined in whole saliva samples from subjects suffering from $A$. actinomycetemcomitansassociated $\mathrm{PD}^{14}$. The output of lactoferrin was not significantly different in healthy and diseased subjects although a higher iron-saturation of lactoferrin in subjects with the disease was described.

Lactoferrin expression was examined in whole and parotid saliva from individuals with localized aggressive periodontitis and compared to age, gender-, and race-matched controls ${ }^{10}$. It was reported that whole saliva from the test group had higher levels of the glycoprotein although the levels of bound iron were significantly reduced, suggesting that lactoferrin from individuals with localized aggressive periodontitis might interfere in the disease progression.

In the present study, it was observed that individuals with or without periodontitis exhibited a similar pattern of lactoferrin secretion (Figure 1B). Comparison of expression among groups revealed that under non-stimulated conditions individuals without periodontitis (CG) showed the highest expression of lactoferrin (Figure 2B) whereas under stimulated conditions individuals with periodontitis (APG and CPG) exhibited a more intense immunoreactive signal (Figure 2D).

The higher expression of this glycoprotein 
in individuals with aggressive periodontitis is in agreement with a previous report ${ }^{10}$, but is in contrast to others ${ }^{14,32}$. The differences in these two studies from the present could be at least in part explained by variations in samples that were analyzed (i.e. SMSL versus whole saliva) since lactoferrin derived from the gingival crevicular fluid will be present in samples from whole saliva.

The increase in the amount of lactoferrin in stimulated samples from SMSL of individuals with generalized chronic periodontitis (Figure 2D) found in the present study is in contrast to another report ${ }^{21}$ in which the levels of lactoferrin in samples from non-stimulated whole saliva were higher than in samples of stimulated whole saliva. Again, the differences in this study from the present could also be in part explained by SMSL versus whole saliva.

Exclusion of individuals that had aggressive or chronic periodontitis and received periodontal treatment during the last year had a direct impact in our sample size, since it was difficult to select individuals that met this specific criterion. Nevertheless, this is the first study that compares the expression of salivary proteins among individuals with aggressive and chronic periodontitis at the same time, and that evaluates the influence of stimulation over time in the expression of MG2 and lactoferrin in individuals with periodontitis. Analysis of these salivary constituents appears to have potential to be used as markers for aggressive or chronic periodontitis, and since both MG2 and lactoferrin possess antimicrobial activity, specific domains could be considered as possible candidates for the development of new drugs that in principle could be of benefit to individuals with periodontitis.

The biological plausibility of the differences observed in SMSL physiology in our study could be derived by periodontal bacterial components triggering the host-immune response, and causing inflammation and activation of pro-inflammatory mediators. It has been described that these molecules traveling via blood to other organs and tissues might influence a variety of mechanisms ${ }^{1}$. It is important to mention that the observed results show that future studies should purposely collect non-stimulated and/or stimulated saliva samples since protein expression under these circumstances varies. The reduced expression of these glycoproteins observed in APG and CPG particularly under non-stimulated conditions, decrease the oral cavity innate defense mechanisms provided by these salivary components in these individuals.

\section{CONCLUSIONS}

It remains unclear to what extent the reduced output of MG2 and lactoferrin might impact the etiopathogenesis of aggressive and chronic periodontitis. However, the data suggest an active role of these glycoproteins in the innate immune regulation of periodontal bacterial colonization and disease progression.

\section{REFERENCES}

1- Amar S, Han X. The impact of periodontal infection on systemic diseases. Med Sci Mon. 2003;9(12):291-9.

2- American Academy of Periodontology. Parameter on aggressive periodontitis. J Periodontol. 2000;71(5):867-9.

3- American Academy of Periodontology. Parameter on chronic periodontitis with advanced loss of periodontal support. J Periodontol. 2000;71(5):856-8.

4- American Academy of Periodontology. Parameter on chronic periodontitis with slight to moderate loss of periodontal support. J Periodontol. 2000;71(5):853-5.

5- Armitage GC. Development of a classification system for periodontal diseases and conditions. Ann Periodontol. $1999 ; 4(6): 1-6$.

6- Armitage GC, Research, Science and Therapy Committee of the American Academy of Periodontology. Diagnosis of periodontal diseases. J Periodontol. 2003;74(8):1237-47.

7- Becerra L, Soares RV, Bruno LS, Siqueira CC, Oppenheim FG, Offner GD, et al. Patterns of secretion of mucins and non-mucin glycoproteins in human submandibular/sublingual secretion. Arch Oral Biol. 2003;48(2):147-54.

8- Biesbrock AR, Reddy MS, Levine MJ. Interaction of a salivary mucin-secretory immunoglobulin: a complex with mucosal pathogens. Infect Immun. 1991;59(10):3492-7.

9- Corraini P, Pannuti CM, Pustiglioni AN, Romito GA, Pustiglioni FE. Risk indicators for aggressive periodontitis in an untreated isolated young population from Brazil. Braz Oral Res. 2009;23(2):209-15. 10 - Fine DH, Furgang D, Beydouin F. Lactoferrin iron levels are reduced in saliva of patients with localized aggressive periodontitis. J Periodontol. 2002;73(6):624-30.

11- Fisher SJ, Prakobphol A, Kajisa L, Murray PA. External radiolabelling of components of pellicle on human enamel and cementum. Arch Oral Biol. 1987;32(7):509-17.

12- Flemmig TF. Periodontitis. Ann Periodontol. 1999;4(1):32-8.

13- Genco RJ. Current view of risk factors for periodontal diseases. J Periodontol. 1996;67(10):1041-9.

14- Groenink J, Walgreen-Weterings E, Nazmi K, Bolscher JG, Veerman EC, Van Winkelhoff AJ, et al. Salivary lactoferrin and low-Mr mucin MG2 in Actinobacillus actinomycetemcomitansassociated periodontitis. J Clin Periodontol. 1999;26(5):269-75. 15- Hägewald S, Bernimoulin JP, Köttgen E, Kage A. Salivary IgA subclasses and bacteria-reactive IgA in patients with aggressive periodontitis. J Periodontol Res. 2002;37(5):333-9.

16- Henskens YM, Van Den Keijbus PA, Veerman EC, Van Der Weijden GA, Timmerman MF, Snoek CM, et al. Protein composition of whole and parotid saliva in healthy and periodontitis subjects. Determination of cystatins, albumin, amylase and IgA. J Periodontal Res. 1996;31(1):57-65.

17- Henskens YM, Veerman EC, Mantel MS, Van Der Velden U, Nieuw Amerongen AV. Cystatins $S$ and $C$ in human whole saliva and in glandular saliva in periodontal health and disease. J Dent Res. $1994 ; 73(10): 1606-14$.

18- Ito T, Komiya-Ito A, Arataki T, Furuya Y, Yajima Y, Yamada S, et al. Relationship between antimicrobial protein levels in whole saliva and periodontitis. J Periodontol. 2008;79(2):316-22.

19- Jalil RA, Ashley FP, Wilson RF, Wagaiyu EG. Concentrations of thiocyanate, hypothiocyanite, "free" and "total" lysozyme, lactoferrin and secretory IgA in resting and stimulated whole saliva of children aged 12-14 years and the relationship with plaque accumulation and gingivitis. J Periodontal Res. 1993;28(2):130-6. 
20- Jensen JL, Lamkin MS, Oppenheim FG. Adsorption of human salivary proteins to hydroxyapatite: a comparison between whole saliva and glandular salivary secretions. J Dent Res. 1992;71(9):1569-76.

21- Jentsch H, Sievert Y, Gocke R. Lactoferrin and other markers from gingival crevicular fluid and saliva before and after periodontal treatment. J Clin Periodontol. 2004;31(7):511-4.

22- Kaufman E, Lamster IB. Analysis of saliva for periodontal diagnosis. A review. J Clin Periodontol. 2000;27(7):453-65.

23- Ligtenberg AJ, Walgreen-Weterings E, Veerman EC, De Soet JJ, De Graaff J, Amerongen AV. Influence of saliva on aggregation and adherence of Streptococcus gordonii HG 222. Infect Immun. 1992;60(9):3878-84.

24- Liu B, Rayment SA, Gyurko C, Oppenheim FG, Offner GD, Troxler RF. The recombinant $\mathrm{N}$-terminal region of human salivary mucin MG2 (MUC7) contains a binding domain for oral Streptococci and exhibits candidacidal activity. Biochem J. 2000;345(3):55764.

25- Liu B, Rayment SA, Oppenheim FG, Troxler RF. Isolation of human salivary mucin MG2 by a novel method and characterization of its interactions with oral bacteria. Arch Biochem Biophys. 1999;364(2):286-93

26- Liu B, Rayment SA, Soares RV, Oppenheim FG, Offner GD, Fives-Taylor $P$, et al. Interaction of human salivary mucin MG2, its recombinant $\mathrm{N}$-terminal region and a synthetic peptide with Actinobacillus actinomycetemcomitans. J Periodontal Res. 2002;37(6):416-24.

27- Mukherjee S, Crawford JM, McClear N, Tsang A. A longitudinal study of unsaturated iron-binding capacity and lactoferrin in nonstimulated parotid saliva. Biol Trace Elem Res. 1997;57(1):1-8.
28- Queiroz AC, Taba M Jr, O'Connell PA, Nóbrega PB, Costa $\mathrm{PP}$, Kawata VK, et al. Inflammation markers in healthy and periodontitis patients: a preliminary data screening. Braz Dent J. $2008 ; 19(1): 3-8$.

29- Rayment SA, Liu B, Offner GD, Oppenheim FG, Troxler RF. Immunoquantification of human salivary mucins MG1 and MG2 in stimulated whole saliva: factors influencing mucin levels. J Dent Res. 2000;79(10):1765-72.

30- Scannapieco FA. Saliva-bacterium interactions in oral microbial ecology. Crit Rev Oral Biol Med. 1994;5(3-4):203-48.

31- Stinson MW, Levine MJ. Modulation of intergeneric adhesion of oral bacteria by human saliva. Crit Rev Oral Biol Med. 1993;4(34):309-14.

32- Suomalainen K, Saxén L, Vilja P, Tenovuo J. Peroxidases, lactoferrin and lysozyme in peripheral blood neutrophils, gingival crevicular fluid and whole saliva of patients with localized juvenile periodontitis. Oral Dis. 1996;2(2);129-34.

33- Tonetti MS, Mombelli A. Early-onset periodontitis. Ann Periodontol. 1999;4(1):39-53.

34- Van Dyke TE. The etiology and pathogenesis of periodontitis revisited. J Appl Oral Sci. 2009;17(1):pii :S1678-77572009000100001.

35- Weinberg ED. Human lactoferrin: a novel therapeutic with broad spectrum potential. J Pharm Pharmacol. 2001;53(10):130310.

36- Yamauchi K, Tomita M, Giehl TJ, Ellison RT $3^{\text {rd }}$. Antibacterial activity of lactoferrin and a pepsin-derived lactoferrin peptide fragment. Infect Immun. 1993;61(2):719-28. 